DIGITALCOMMONS

—@WAYNESTATE -
Journal of Modern Applied Statistical Methods

Volume 6 | Issue 1

$5-1-2007$

\title{
Estimation of Risk for Developing Cardiac Problem in Patients of Type 2 Diabetes as Obtained by the Technique of Density Estimation
}

\author{
Ajit Mukherjee \\ Division of Reproductive Health and Nutrition, Indian Counsel of Medical Research, New Delhi, India \\ Ajit Mathur \\ Division of Reproductive Health and Nutrition, Indian Counsel of Medical Research, New Delhi, India \\ Rakesh Mittal \\ Division of Reproductive Health and Nutrition, Indian Counsel of Medical Research, New Delhi, India
}

Follow this and additional works at: http://digitalcommons.wayne.edu/jmasm

Part of the Applied Statistics Commons, Social and Behavioral Sciences Commons, and the Statistical Theory Commons

\section{Recommended Citation}

Mukherjee, Ajit; Mathur, Ajit; and Mittal, Rakesh (2007) "Estimation of Risk for Developing Cardiac Problem in Patients of Type 2 Diabetes as Obtained by the Technique of Density Estimation," Journal of Modern Applied Statistical Methods: Vol. 6 : Iss. 1 , Article 29. DOI: $10.22237 /$ jmasm/1177993680

Available at: http://digitalcommons.wayne.edu/jmasm/vol6/iss1/29

This Regular Article is brought to you for free and open access by the Open Access Journals at DigitalCommons@WayneState. It has been accepted for inclusion in Journal of Modern Applied Statistical Methods by an authorized editor of DigitalCommons@WayneState. 


\title{
Estimation of Risk for Developing Cardiac Problem in Patients of Type 2 Diabetes as Obtained by the Technique of Density Estimation
}

\author{
Ajit Mukherjee \\ Ajit Mathur \\ Rakesh Mittal \\ Division of Reproductive Health and Nutrition, Indian Council of Medical Research \\ New Delhi, India
}

High levels of cholesterol and triglyceride are known to be strongly associated with development of cardiac problem in patients of type 2 diabetes. In a hospital-based study, patients showing ECG positive were compared with those who were not. The observations on cholesterol and triglyceride were considered for estimation of risk for developing the cardiac problem. The technique of density estimation employing Epanechnikov kernel was used for estimating bivariate probability density functions with respect to observations on cholesterol and triglyceride of the two groups. Using the odds form of Bayes' rule, the estimates of posterior odds were computed.

Key words: Density estimation, kernel, logistic regression, probability density function.

\section{Introduction}

The technique of Density Estimation is a nonparametric approach and involves no assumptions as it deals directly with the experimental data. The method of density estimation describes the probability distribution of people with respect to the parameter under investigation. This technique has found favour with many applied statisticians in the past. Scott, Gotto, Cole, and Gorry (1978) used density

Ajit Mukherjee, Ph.D. in Mathematical Statistics, is Deputy Director General in Division of Reproductive Health and Nutrition, Indian Council of Medical Research, Ansari Nagar, Post Box 4911, New Delhi-110029, India. He has over 30 publications to his credit in both national and international journals and is the holder of a prestigious National Award Email: mukherjeeajit@hotmail.com. Ajit Mathur, M.Sc., is also Deputy Director General in Division of Reproductive Health and Nutrition. $\mathrm{He}$ has 25 publications to his credit. Email:ajiticmr@hotmail.com. Rakesh Mittal, M. D., is Deputy Director General (Senior Grade), also in Division of Reproductive Health and Nutrition. He has about 30 publications and books to his credit.E-mail:mittrak@hotmail.com estimation for assessing plasma lipids as collateral risk factors in coronary artery disease. Bithell (1990) gave an application of this technique in Geographical Epidemiology. Mukherjee, Kumar, Mittal, and Saxena (2002) used density estimation for estimating risk of developing goiter in an endemic area. Silverman (1986) provided an excellent account of various approaches to Density Estimation in his book.

High levels of cholesterol and triglyceride are known to be strongly associated with development of cardiac problem in patients of type 2 diabetes. However, the extent of risk posed by elevated levels of these two risk factors in patients of type 2 diabetes has not been studied extensively. The present article describes an alternative methodology whereby risk of developing cardiac problem in patients of type 2 diabetes can be estimated using cholesterol and triglyceride as risk factors.

\section{Methodology}

In a hospital-based study conducted by Indian Council of Medical Research in 1989-92, 4637 patients of Non Insulin Dependent Diabetes Mellitus (NIDDM) also known as Type2 Diabetes were enrolled. Various bio-chemical investigations and electrocardiogram (ECG) were carried out at regular intervals. The 311 
patients showing ECG positive and thereby indicating coronary artery disease $(\mathrm{CAD}+)$, formed the first group. The remaining patients numbering 4326 formed the second group. The Epanechnikov kernel, which is known to have $100 \%$ efficiency in terms of mean integrated square error (Silverman, 1986), was employed in the technique of density estimation for estimating probability density functions of the patients falling in the two groups with respect to their cholesterol and triglyceride levels. Using the odds form of Bayes' rule, the estimate of odds ratio (OR) was obtained. A simulation study was undertaken and 100 estimates of OR were generated using the approach of density estimation giving a mean estimated odds ratio and an estimate of standard deviation.

Let the number of patients in the first group be denoted by $N_{C A D+}$ and that in the second group by $N_{N I D}$. Let $\mathrm{x}$ and y in general denote the observations on cholesterol and triglyceride of the patients with $x_{i}$ and $y_{i}$ being the observations on the ith patient. Then the bivariate kernel density estimator for the first group is given by

$$
\begin{aligned}
& \mathrm{f}_{\mathrm{CAD}+} \equiv \mathrm{f}_{\mathrm{CAD}+}(\mathrm{x}, \mathrm{y}) \\
& =\frac{1}{\mathrm{~N}_{\mathrm{CAD}+} \mathrm{h}_{\mathrm{x}} \mathrm{h}_{\mathrm{y}}} \sum_{\mathrm{i}=1}^{\mathrm{N}_{\mathrm{CAD}+}} \mathrm{K}\left(\frac{\mathrm{x}-\mathrm{x}_{\mathrm{i}}}{\mathrm{h}_{\mathrm{x}}}\right) \mathrm{K}\left(\frac{\mathrm{y}-\mathrm{y}_{\mathrm{i}}}{\mathrm{h}_{\mathrm{y}}}\right)
\end{aligned}
$$

the quantities $h_{x}$ and $h_{y}$ are called bandwidths of the function $f_{C A D}+$ and are appropriately chosen.

The function $\mathrm{K}(\mathrm{z})$ which is known as Epanechnikov kernel, is defined as follows:

$$
K(z)=\left\{\begin{array}{l}
\frac{3}{4}\left(1-\frac{1}{5} z^{2}\right) / \sqrt{5}, \text { if }|z|<\sqrt{5} \\
0, \text { otherwise }
\end{array}\right.
$$

Similarly, the Epanechnikov kernel density estimator for the other group namely, $f_{N I D} \equiv f_{N I D}(x, y)$ can also be worked out.

The Likelihood Ratio (LR) will then be given by

$$
L R=\frac{f_{C A D+}}{f_{N I D}} .
$$

Further, the odds form of Bayes' rule states that

$$
O^{\prime}(D)=O(D) * L R
$$

where $O^{\prime}(D)$ is the posterior odds and $O(D)$ is prior odds and is given as $O(D)=\frac{N_{C A D+}}{N_{N I D}}$.

For the present set of data, $N_{C A D+}=311$ and $N_{N I D}=4326$. For estimating $f_{C A D+}$, the optimum values of $h_{x}$ and $h_{y}$ were obtained by objectively starting the process of smoothing with $h_{x}=h_{y}=5$ giving an increment of 5 until $h_{x}=h_{y}=35$. Thereafter, the process of smoothing was continued by giving a unit increment. The estimates of density stabilized with values of $h_{x}$ and $h_{y}$ at 40, 41,42, 43 to five decimal places. Hence, $h_{x}=h_{y}=40$ was accepted as an optimum value of $h_{x}$ and $h_{y}$.

Similarly, the values of $h_{x}$ and $h_{y}$ for estimating $f_{N I D}$ were also obtained to be 40 each. The estimate of probability density function for the first group i.e., $f_{C A D+}$ is as depicted in Figure 1.

Computation of OR

Consider the following transformation

$$
\begin{gathered}
P=\frac{1}{1+e^{-Y}}, \text { where } \\
Y=\beta_{0}+\beta_{1} X_{1}+\beta_{2} X_{2}+---+\beta_{k} X_{k}+\varepsilon .
\end{gathered}
$$

It can be shown that 
Fig.1. Bivariate Probability Density Function of patients with CAD+ at different values of Cholesterol and Triglyceride using Epanechnikov Kernel

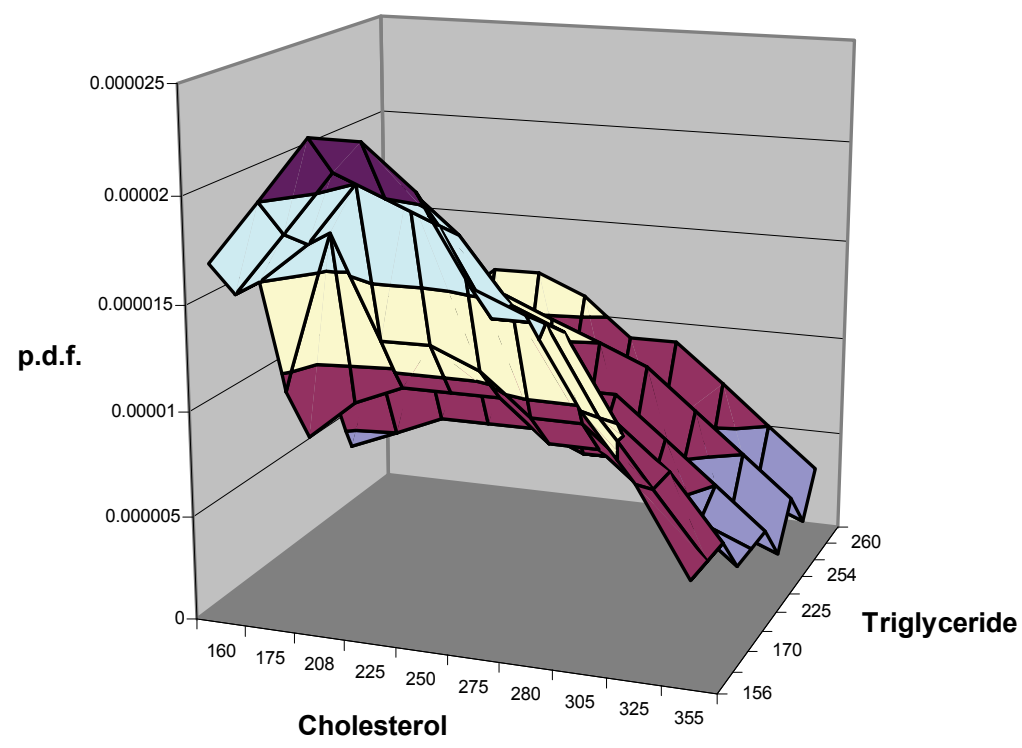

Q0.00002-0.000025 口0.000015-0.00002 口0.00001-0.000015 口0.000005-0.00001 口0-0.000005

$$
\log \left(\frac{P}{1-P}\right)=Y
$$

or

$\log \left(\frac{P}{1-P}\right)=\beta_{0}+\beta_{1} X_{1}+\beta_{2} X_{2}+---+\beta_{k} X_{k}+\mathcal{E}$

Thus $\beta_{i}$ gives average quantum of change in $\log$ (odds) per unit change in $X_{i}, i=1,2, \ldots . ., k$ and $e^{\beta_{i}}$ gives the odds ratio with respect to $X_{i}$ keeping other predictors at constant levels.

\section{Results}

Keeping cholesterol fixed at 250 and varying the value of triglyceride from 209 to 254 , the posterior odds at three pairs of values of cholesterol and triglyceride viz., (250,209), $(250,254)$ and $(250,260)$ were worked out to be respectively $0.0629,0.08047$ and 0.08549 . Thus, keeping cholesterol fixed at 250 and increasing triglyceride by a margin of 45 and 51 units from 209 , led to respectively 1.28 and 1.34 times increase in odds for developing a cardiac problem. Further, considering first two of the above three pairs of values of cholesterol and triglyceride, the following would be obtained:

$$
\beta_{Y}=\frac{\log (0.08047)-\log (0.0629)}{45}
$$

or

$$
\beta_{Y}=0.005474
$$


Therefore $\mathrm{OR}$ is given by $e^{\beta_{Y}}=1.005489$. Drawing a simple random sample of 100 consecutive pairs of values of cholesterol and triglyceride and using the above methodology, 100 estimates of OR were obtained with a mean value of OR as 1.0025 and S.D. of 0.0027 giving $95 \%$ C.I. as $\{1.0020,1.0031\}$. The OR as estimated by Logistic Regression model was 1.0029 with a $95 \%$ C.I. of $\{0.9984,1.0074\}$.

\section{Conclusion}

It is seen that with the technique of density estimation employing Epanechnikov kernel, it is possible to obtain an estimate of the probability density function of the patients of type 2 diabetes falling in the two groups with respect to their cholesterol and triglyceride levels. It has also been demonstrated in the present article, how the posterior odds vary with increasing levels of triglyceride keeping cholesterol at a constant high level, which ultimately led to an estimate of odds ratio (Table 1).

From table 1, it is clear that the estimate of odds ratio as obtained by the method of density estimation is in close proximity to the estimate as obtained by the method of logistic regression. Thus, the risk of developing a cadiac problem can also be alternatively estimated by using the technique of density estimation.
References

Bithell, J. F (1990). An application of density estimation to geographical epidemiology. Statistics in Medicine, 9, 691701.

Hennekens, C. H. \& Burning, J. E. (1987). In (S. L. Mayrent, Ed.) Epidemiology in medicine. Boston, MA: Little, Brown and Co.

Mukherjee, A., Kumar, S., Mittal, R. \& Saxena, N. C. (2002). An application of the technique of density estimation in geographical epidemiology of goitre in the Muzaffarpur district of Bihar state of India. Statistics in Medicine, 21, 2403-2407.

Scott, D. W., Gotto, A. M., Cole, J.S., \& Gorry, G. A. (1978). Plasma lipids as collateral risk factors in coronary artery disease: A study of 371 males with chest pain. Journal of Chronic Diseases, 31, 337-345.

Silverman, B. W (1986). Density estimation for statistics and data analysis. London: Chapman and Hall.

Table 1. Estimates of Odds Ratio as obtained by the application of Epanechnikov kernel in Density Estimation and Logistic Regression

\begin{tabular}{|c|c|c|}
\hline Method & Odds Ratio & 95\% C.I. \\
\hline Logistic Model & 1.0029 & $0.9984-1.0074$ \\
\hline Density Estimation: & & $1.002-1.0031$ \\
\hline Epanechnikov Kernel & 1.0025 & \\
\hline
\end{tabular}

